Recepción: 07/05/2016

Evaluación: 12/05/2016

Aprobación:15/06/2016

Artículo de Revisión

\title{
CONTENIDO Y ALCANCES DEL DERECHO A LA AUTONOMÍA UNIVERSITARIA DESDE UN PUNTO DE VISTA NORMATIVO Y JURISPRUDENCIAL
}

\author{
Wilmer Jahir Sierra Fagua \\ Universidad Pedagógica y Tecnológica de Colombia \\ Grupo de Investigación: Docente Catedrático \\ wsierrafagua@yahoo.com
}

\begin{abstract}
RESUMEN
Este artículo plantea la necesidad de entender el contenido y los verdaderos alcances del Derecho a la autonomía universitaria; se precisa que la información obtenida se auscultó desde una perspectiva interpretativa. Como punto de partida se efectúa un estudio y un análisis sobre la importancia de la Constitución Política de 1991, para la aplicación e interpretación de los derechos en Colombia, hasta llegar al principio de la autonomía universitaria como pilar fundamental de las Universidades.

El resultado de esta investigación determinará si las instituciones universitarias conforme al marco normativo constitucional y legal que las rige, así como desde la interpretación constitucional que sobre el particular se ha efectuado, se erigen como verdaderos centros de pensamiento libres e independientes, en donde están proscritas las imposiciones y/o presiones externas que se encaminen a limitar, entorpecer o impedir bajo cualquier forma el desarrollo pleno de su autonomía.

Con el examen de las interpretaciones efectuadas a la Carta Política por la Corte Constitucional, se evidenciará que la autonomía universitaria no implica en modo alguno un total margen de discrecionalidad o libertad absoluta y desmedida, en tanto es claro que se encuentra sujeta a varios límites que le proscriben cierto tipo de acciones, pero que a su vez le señalan claramente cuál es el horizonte que se les quiso asentir.
\end{abstract}

Palabras clave: Derechos humanos, Constitución, autonomía educativa, libertad de enseñanza, universidad. 


\title{
CONTENT AND SCOPE OF THE RIGHTS OF UNIVERSITY AUTONOMY FROM A REGULATORY AND JURISPRUDENTIAL PERSPECTIVE
}

\begin{abstract}
This article reflects upon the need to understand the content and true scope of the Rights of University Autonomy, which requires that the information obtained is looked at from an interpretive perspective.

The primary point of the study is an analyses of the importance of the political Constitution of 1991 to the application and interpretation of Rights in Colombia relating to the principles of University Autonomy as a fundamental pillar of universities.

The result of this investigation will determine if the university institutions are in accordance with the constitutional and legal governing framework, as well as the constitutional interpretation that has been made on the particular stand of being true centers of free and independent thought, and whether they impose bans or external pressures that would limit, interfere with, or prevent the full development of institutional autonomy in any form.

Examination of interpretations made to the Political Charter by the Constitutional Court show that university autonomy does not in any way imply a total margin of discretion or absolute and unrestrained liberty so clear that it is subject to various limits proscribing certain types of actions, but which in turn clearly indicate what they want to achieve.
\end{abstract}

Key Words: Human Rights, Constitution, Educational Autonomy, freedom of education, university.

\section{CONTEÚDO E ÂMBITO DO DIREITO À AUTONOMIA UNIVERSITÁRIA DE UM PONTO DE VISTA REGULAMENTAR DA JURISPRUDÊNCIA}

\begin{abstract}
RESUMO
Este artigo sugere a necessidade de compreender o conteúdo e o verdadeiro alcance do direito à autonomia universitária; ele afirma que as informações obtidas a partir de uma perspectiva interpretativa auscultado.

Como ponto de partida se efetua um estudo e uma análise da importância da Constituição política de 1991, para a aplicação e interpretação dos direitos na Colômbia, até chegar ao princípio da autonomia universitária como um pilar fundamental das universidades.
\end{abstract}


O resultado desta investigação determinará se as instituições universitárias conformidade com oo quadro constitucional e de regulamentação legal que os governa, bem como a partir da interpretação constitucional sobre o assunto foi feito, se apresentam como verdadeiros centros de pensamento livre e independente, onde são proibidos as imposições e /ou pressões externas que são encaminhadas para limitar, dificultar ou impedir de qualquer forma o pleno desenvolvimento de sua autonomia. Com o exame das interpretações feitas à carta política pelo Tribunal Constitucional, poderá evidenciar que a autonomia universitária não implica de forma alguma uma total margen de discrição ou liberdade absoluta e desmedida, embora seja claro que está sujeita a vários limites que proscrevem certos tipos de tais ações, mas que por sua vez deve indicar claramente qual é o horizonte que se quis assentir.

Palavras-Chave: Direitos Humanos, Constituição, autonomia educativa, liberdade de ensino, universidade.

\section{INTRODUCCIÓN}

Adentrándose en el estudio de los Derechos se encuentra de forma implícita el de la autonomía universitaria. Derecho que suscita cuestionamientos y debates cuando de detallar sus alcances se trata. Previo a abordar el tema de autonomía universitaria, planteo un análisis sobre la importancia de los derechos en Colombia, a partir de la Constitución política de 1991.

Abordando luego el tema desde dos puntos de vista, el primero, a través de una exposición de su marco normativo, en segunda instancia, destacando los alcances que la Corte Constitucional a través de su copiosa jurisprudencia le ha otorgado.

Bajo estos criterios, la reflexión se plantea en dos sentidos:

1. Autonomía universitaria entendida como autorregulación filosófica y como auto organización interna.

2. Autonomía universitaria e importancia para el Estado y límites de sujeción que se le han impuesto.

\section{UN BREVE ACERCAMIENTO A LA IMPORTANCIA DE LOS DERECHOS EN COLOMBIA}

Sobre el particular, sea lo primero manifestar que a partir del año 1991, la historia de los derechos en Colombia dio un giro de 180 grados, ello aconteció con el advenimiento de la Constitución de 1991. El nuevo texto que vino a reemplazar la Constitución más larga y duradera en la historia constitucional colombiana, la de 1886, se llevó a cabo dentro de un proceso de coyuntura 
y de cambio político gestado como una respuesta a las situaciones de violencia política y social que desde comienzo de los años ochenta se vivió en Colombia, valga recordar que en esa época se presentó una fuerte lucha entre las guerrillas y el Estado, marcada por una evidente influencia de los recursos del narcotráfico, lo que llevó a una gran "descomposición social”, que a su vez desencadenó en una sociedad con pérdida de valores generadores de articulación, y con pocos referentes colectivos en torno a cuales organizarse ${ }^{1}$ (Ladrón de Guevara, 2002).

La Asamblea Nacional Constituyente de 1991, fue el evento más representativo de la democracia en Colombia, en la medida que en ella concurrió una gran pluralidad nacional -representantes indígenas, organizaciones religiosas, movimientos guerrilleros, como el M-19, la Unión Patriótica, el Movimiento Quintín Lame-, dicha Asamblea fue precedida de acuerdos de paz con fracciones del movimiento guerrillero; acuerdos que continuaron durante la Asamblea incorporando más representantes a la negociación política ${ }^{2}$ (Restrepo Botero, 1992).

Existieron circunstancias claves que posibilitaron el cambio constitucional, una, el cansancio con la espera de la insurrección armada, propiciado por el colapso de los países del socialismo real, situación que dejó -en principiosin perspectiva revolucionaria al movimiento guerrillero colombiano, dos, el convencimiento de los narcotraficantes, frente al hecho de haber entendido que no habían logrado doblegar por la vía de la violencia a los representantes políticos del sistema, circunstancia, que los llevó a gestionar y concretar la prohibición constitucional de la extradición ${ }^{3}$ (Restrepo Botero, 1992).

Debe destacarse que en el curso de la nueva Constitución hubo dos consultas populares, una informal -denominada séptima papeleta- que llevó a su vez a una consulta formal sobre la convocatoria a una Asamblea Nacional Constituyente, hubo dos decretos de estado de sitio, y dos fallos favorables de la Corte Suprema de Justicia que le dieron vía libre a la formación de la nueva Constitución. Finalmente, se dieron tres procesos electorales que pretendieron solucionar el problema de la legitimidad-legalidad-, del proceso constituyente que se pretendía engendrar ${ }^{4}$ (Dávila Ladrón de Guevara).

Al adentrarse en el contenido normativo de la Constitución de 1991, se encuentra que desde el artículo primero se estableció un modelo denominado

\footnotetext{
1. Andrés Dávila Ladrón de Guevara, Democracia pactada: El frente Nacional y el proceso constituyente del 91 (Bogotá D.C.: Alfaomega Grupo Editor, 2002), 164-165.

2. Darío Indalecio Restrepo Botero, Descentralización y Neoliberalismo Balance de un proceso (Bogotá D.C, Fondo Editorial CEIR- Realidad Municipal, 1992), 232-233.

3. Restrepo Botero, Descentralización, 234, 235, 236.

4. Dávila Ladrón de Guevara, Democracia pactada, 172 y 173.
} 
Estado social de derecho 5 (Quinche Ramírez, 2010), el que repercute en tres pilares fundamentales, a saber:

- El principio de legalidad, según el cual tanto los gobernantes como los ciudadanos nos encontramos en un mismo plano de igualdad y por ende sujetos a un solo marco jurídico, siendo deber del Estado en todas sus ramas, plasmar las regulaciones que han de regir los destinos de la sociedad, con base en ello es posible afirmar que todo lo que no está proscrito por normas previamente establecidas, luego no podrá ser reprochado por ninguna autoridad Estatal.

- El principio de división de poderes, con el que se persigue que cada una de las diferentes competencias que existen dentro del Estado se repartan entre diferentes autoridades, las cuales gozan de plena autonomía para desplegarlas, a su vez, entre cada una de éstas se ejerce un estricto control encaminado a evitar que alguna de ellas adquiera un poder mayoritario o un control superior, lo que conlleva a que entre todas se equilibren de forma armónica.

- El Reconocimiento explícito de los derechos, así como la obligación del Estado de proceder a hacerlos efectivos, de materializarlos.

En relación con el último tópico, resulta trascendental adentrarse el contenido del artículo 2 Constitucional, en tanto dispone de forma abierta, precisa y contundente para qué y por qué se instituyó el Estado colombiano. En efecto, allí se estableció lo siguiente:

i. El Estado colombiano tiene como fines esenciales, entre otros, los de servir a la comunidad, promover la prosperidad general y garantizar la efectividad de los principios, derechos y deberes consagrados en la Constitución;

ii. Asimismo, las autoridades de la República están instituidas para proteger a todas las personas residentes en Colombia, en su vida, honra, bienes, creencias, y demás derechos y libertades, y para asegurar el cumplimiento de los deberes sociales del Estado y de los particulares.

Adicionalmente, debe dejarse de presente que el nuevo texto constitucional revolucionó la historia de los derechos hasta ahora conocida en Colombia, al establecer a partir de su artículo 4, que sería norma de normas, es decir, que estaría por encima de la ley, y que en caso de incompatibilidad entre la Constitución y una ley de cualquier rango o nivel, la primera siempre primaria,

5. Manuel Fernando Quinche Ramírez, Derecho Constitucional Colombiano, de la Carta de 1991 y sus reformas, Cuarta Edición (Bogotá D.C.: Ediciones Doctrina y Ley Ltda., 2010), 52 a 55. 
siendo obligación de los funcionarios del Estado y de los jueces la de inaplicar la norma contradictoria.

Del mismo modo, creó la Corte Constitucional confiándole la primordial función de guardar la integridad y supremacía de la Constitución, encargándola de conocer entre otros, de las demandas de inconstitucionalidad -esto es, cuando una norma contraría el contenido de cualquiera de los preceptos de la Constitución-, presentadas por los ciudadanos en contra de los actos reformatorios de la Constitución, y de las leyes, así como de revisar las decisiones judiciales relacionadas con la acción de tutela de los derechos constitucionales (Artículo 241).

Dentro del Título II se establecieron parte de los derechos que en adelante protegerían a todos los ciudadanos dentro del territorio colombiano, así, del artículo 11 al 41, se enlistaron los fundamentales, del 42 al 77, los derechos sociales, económicos y culturales; finalmente, del artículo 78 al 82, los derechos colectivos y del medio ambiente. Importa precisar que todo el conglomerado de derechos antes mencionados corresponde a lo que doctrinal y jurídicamente se conoce como derechos humanos ${ }^{6}$ (Uprimny Yepes, Uprimny Yepes, Parra Vera. 2006).

Pero quizás uno de los cambios más significativos consistió en establecer las acciones o procedimientos a través de los cuales acudiendo ante los jueces de la República, sería posible buscar del Estado e incluso de los particulares, el cumplimiento de todo ese amplio catálogo de derechos. Así, se creó la acción de tutela (Artículo 86) enfocada a buscar la protección de los derechos fundamentales; la acción de cumplimiento que le otorga "a toda persona, natural o jurídica, e incluso a los servidores públicos, la posibilidad de acudir ante la autoridad judicial para exigir la realización o el cumplimiento del deber que surge de la ley o del acto administrativo y que es omitido por la autoridad, o el particular cuando asume este carácter"7 (Corte Constitucional, C157-98) (Artículo 87); en último término, las acciones populares y de grupo, para la protección de los derechos colectivos y la indemnización de los daños causados a un número plural de personas (Artículo 88).

Conforme a lo anterior, y a modo de conclusión respecto de este capítulo, debe manifestarse que en aplicación del modelo de Estado social de derecho prescrito en la Carta Política de 1991, sin excepción, todas las autoridades que actúen en su nombre y representación, están obligadas a respetar y hacer efectivos cada uno de los derechos que a lo largo y ancho de su texto se Encuentran plasmados, en caso de no hacerlo, los ciudadanos contamos con

6. Rodrigo Uprimny Yepes, Inés Margarita Uprimny Yepes, Oscar Parra Vera, Derechos humanos y derecho internacional humanitario, módulo de autoformación (Bogotá: Fundación Social, Consejo Superior de la Judicatura, Escuela Judicial “Rodrigo Lara Bonilla”, enero 2006), 16 a 20.

7. Corte Constitucional, sentencia C- 157 de 1998, Magistrados Ponentes: Dr. Antonio Barrera Carbonell y Dr. Hernando Herrera Vergara. 
los mecanismos jurídicos idóneos para demandar ante los jueces su efectivo cumplimiento.

\section{EL PRINCIPIO DE LA AUTONOMÍA UNIVERSITARIA COMO PILAR FUNDAMENTAL DE LAS UNIVERSIDADES COLOMBIANAS, CONTENIDO NORMATIVO E INTERPRETACIÓN JURISPRUDENCIAL OTORGADA}

La autonomía universitaria se estableció como un derecho social, económico y cultural en el artículo 69 de la Constitución Política de 1991, su contenido básico se puede desglosar de la siguiente forma: $i$. Las universidades podrán darse sus directivas y regirse por sus propios estatutos; ii. Las universidades del Estado deben gozar de un régimen especial, dado por la ley; iii. El Estado está obligado a fortalecer la investigación científica en las universidades oficiales y privadas y ofrecer las condiciones especiales para su desarrollo; iv. El Estado debe facilitar mecanismos financieros que hagan posible el acceso de todas las personas aptas a la educación superior.

Entre tanto, su desarrollo legal se dio a través de la Ley 30 de 1992, reconociéndole a la universidades en elartículo 29, el derecho a darse y modificar sus estatutos, designar sus autoridades académicas y administrativas, crear, organizar y desarrollar sus programas académicos, definir y organizar sus labores formativas, académicas, docentes, científicas y culturales, otorgar los títulos correspondientes, seleccionar a sus profesores, admitir a sus alumnos y adoptar sus correspondientes regímenes, y establecer, arbitrar y aplicar sus recursos para el cumplimiento de su misión social y de función institucional.

Aunado a lo anterior, en el artículo 57, se determinaron los siguientes parámetros frente a la forma como las universidades estarían organizadas, esto es:

i. Como entes universitarios autónomos, con régimen especialy vinculados al Ministerio de Educación Nacional en lo que se refiere a las políticas y la planeación del sector educativo; con personería jurídica, autonomía académica, administrativa y financiera, patrimonio independiente y la facultad de elaborar y manejar su presupuesto de acuerdo con las funciones que le corresponden;

ii. Finalmente se determinó que el carácter especial otorgado a las universidades estatales u oficiales, comprendería la organización y elección de directivas, del personal docente y administrativo, el sistema de las universidades estatales u oficiales, el régimen financiero, el régimen de contratación y control fiscal y su propia seguridad social en salud.

Llegados a este punto, conviene indicar que la Corte Constitucional como máxima guardiana de nuestra Constitución, y por ende como la intérprete más autorizada frente a sus contenidos, a través de sus sentencias le ha dado al 
derecho en análisis un contenido bastante extenso, que va desde fundamentar su importancia para el Estado colombiano, hasta establecer los límites a los que se encuentra sujeto.

Bajo ese contexto ha determinado que la autonomía universitaria es una garantía institucional que busca legitimar la capacidad de autorregulación y autogestión asignada a las instituciones públicas y privadas encargadas de la prestación del servicio público de educación superior, orientándose a garantizar la libertad de cátedra e investigación, "evitando que el acceso a la formación académica e ideológica de los educandos pueda verse limitado o influido indebidamente por los órganos políticos del Estado en quienes reposa el ejercicio del poder público"8.

La Corte también ha justificado que la autonomía universitaria se erige para garantizarle a la sociedad un espacio libre e independiente "en las áreas del conocimiento, la investigación científica, la tecnología y la creatividad; espacio (...) delimitado tan sólo por el respeto a los principios de equidad, justicia y pluralismo"9.

Lo anterior dicho en otras palabras, las instituciones universitarias se erigen como verdaderos centros de pensamiento libres $e$ independientes, en donde están proscritas las imposiciones y/o presiones externas que se encaminen a limitar, entorpecer o impedir bajo cualquier forma el desarrollo pleno de ese cometido.

Todas las justificaciones anteriores llevaron a la Corte a escindir el principio en estudio bajo dos grandes líneas, por un lado, una llamada autorregulación filosófica, que se enmarca en la libertad de pensamiento y pluralismo ideológico, por otro, en la autodeterminación administrativa ${ }^{\mathbf{1 0}}$, que se circunscribe a la forma como se organizan internamente los centros universitarios, atendiendo a las premisas que desde el artículo 69 de la Constitución y artículos 29 y 57 de la Ley 30 de 1992, se le han otorgado.

Es preciso indicar que la mentada Corporación judicial al estudiar sistemáticamente la Constitución Nacional, encontró que la autonomía universitaria no es ilimitada, ya que se encuentra sujeta a las siguientes restricciones ${ }^{\mathbf{1 1}}$ :

i. La facultad reconocida al Estado para regular y ejercer la suprema inspección y vigilancia de la educación (C.P. art. 67), la que en todo caso

8. Sentencia C-1435 de 2000, con ponencia de la magistrada Cristina Pardo Schlesinger. Objeciones presidenciales al Proyecto de ley No. 118 de 1999 Cámara - 236 de 2000 Senado "Por la cual se modifica el inciso tercero del artículo 57 de la ley 30 de 1992".

9. Sentencia C-1435 de 2000.

10. Sentencia C-1435 de 2000.

11. Sentencia C-1435 de 2000. 
debe entenderse como un control limitado que no puede menoscabar su autonomía, a modo de ejemplo se pueden extraer las consideraciones de la Sentencia C-926 de 200512, en la que indicó que esa facultad de control se traduce en una labor de supervisión sobre la calidad de la instrucción, el manejo ordenado de la actividad institucional y la observancia de las grandes directrices de la política educativa, sin que ello le permita al Estado ejercer observaciones o registros sobre los nombramientos de docentes, o personal administrativo, y menos aún de examinar las tendencias filosóficas o culturales que animan las actividades educativas o de investigación de los centros universitarios.

ii. La competencia atribuida al legislador para expedir las disposiciones generales con arreglo a las cuales las universidades pueden darse sus directivas y regirse por sus propios estatutos (C.P. art. 69);

iii. La facultad de configuración legislativa para expedir las leyes que regirán la prestación efectiva de los servicios públicos (C.P. art. 15023). Respecto de estas dos últimas competencias, debe dejarse de presente que el legislador no tiene facultades explícitas ni implícitas para conferir al gobierno la facultad de limitar la autonomía financiera de las universidades, así, por ejemplo, en la sentencia C-507 de 200813, al estudiar una demanda de inconstitucionalidad en contra del Plan Nacional de Desarrollo 2006-2010, explicitó que el legislador y el ejecutivo tienen vedado intervenir en la destinación de las transferencias que se le hacen a las universidades, so pena de vulnerar su autodeterminación.

iv. Aunado a lo expuesto, no puede dejarse de manifestar que la autonomía universitaria "no es soberanía educativa, pues si bien otorga un margen amplio de discrecionalidad a la institución superior, le impide la arbitrariedad"14. El anterior precepto ha llevado a imponerle uno de los límites más certeros y estudiados a lo largo de las sentencias de la Corte Constitucional; el respeto por el ejercicio de los derechos fundamentales (C.P. arts. 11 y sig.); en este sentido, y a modo de ejemplo se trae a colación la sentencia T-691 de $2012^{15}$, en la que se analizó una acción de tutela interpuesta por un estudiante en contra de la Universidad Distrital Francisco José de Caldas - Facultad Tecnológica, al considerar que había sido discriminado por un docente en razón a su etnia.

12. Con ponencia del Magistrado Dr. Jaime Córdoba Triviño.

13. Con ponencia del Magistrado Dr. Jaime Córdoba Triviño.

14. Sentencia C-008 de 2001, Demanda de inconstitucionalidad contra el Artículo 103 de la Ley 30 de 1992, Actores: James Fernández Cardozo y Carlos Olmedo Arias Rey, Magistrado Ponente: Dr. Alvaro Tafur Galvis.

15. Con ponencia de la Magistrada, Dra. María Victoria Calle Correa. 
Allí la Corte sostuvo que la autonomía universitaria es un derecho íntimamente ligado con la libertad de cátedra, no obstante, en casos de discriminación racial que lleva inmersa la vulneración de derechos fundamentales, es obligación de los entes universitarios tomar todas la medidas necesarias a fin de conjurarlos, del mismo modo, determinó que:

La Universidad desconoció los derechos a la igualdad, a no ser discriminado y al debido proceso de Heiler Yesid Ledezma Leudo, una persona que, en su calidad de estudiante, solicitó que se adoptaran medidas de protección ante un acto de discriminación. Es irrazonable pretender que el ejercicio del derecho de autonomía universitaria, se pueda usar para querer justificar un acto prohibido constitucionalmente: no adelantar el trámite de una solicitud presentada, observando las reglas mínimas de un debido proceso constitucional. Esto es especialmente grave si la institución adelantó una investigación informal que (i) comprobó que en efecto sí se había sometido al estudiante a un escenario de discriminación, y (ii) que se requería tomar medidas de protección. Por tanto, la Universidad Distrital Francisco José de Caldas dejó de proteger los derechos a la igualdad y a la no discriminación de Heiler Yesid Ledezma Leudo.

\section{Conclusiones}

Recapitulando, y a modo de conclusiones generales frente a este capítulo, es dable manifestar que la autonomía universitaria es un derecho del que gozan todas las instituciones encargadas de brindar el servicio público de educación superior. Este derecho tiene un doble sentido, por un lado frente a su filosofía y libertad de pensamiento, por otro frente a la forma como han de autogobernarse internamente.

La autonomía universitaria propende por proscribir cualquier tipo de intervenciones o presiones externas que desde el Estado a través de sus diferentes instituciones se pretendan efectuar sobre los entes universitarios, evitando con ello que se coarte de alguna forma cada uno de los pilares fundamentales que componen en esencia el mencionado derecho.

Con todo y lo anterior, la autonomía universitaria no implica en modo alguno un total margen de discrecionalidad o libertad absoluta y desmedida, en tanto es claro que se encuentra sujeta a unos infranqueables límites impuestos desde la misma Constitución Política, y desarrollados por la Corte Constitucional a través de sus prolijas sentencias. 


\section{REFERENCIAS BIBLIOGRÁFICAS}

Bernal Pulido, Carlos. El Derecho de los Derechos, escritos sobre la aplicación de los derechos fundamentales, quinta reimpresión. Bogotá D.C.: Universidad Externado de Colombia, 2008.

Bonorino, Pablo Raúl; Peña Ayaso, Jairo Iván. Libro Filosofía del Derecho. Escuela Judicial Rodrigo Lara Bonilla,

Dávila Ladrón de Guevara, Andrés. Democracia pactada: el Frente Nacional y el proceso constituyente del 91, Bogotá D.C.: Alfaomega Grupo Editor, 2002.

Quinche Ramírez, Manuel Fernando. Derecho Constitucional Colombiano, de la Carta de 1991 y sus reformas. Cuarta Edición. Bogotá D.C.: Ediciones Doctrina y Ley Ltda, 2010.

Restrepo Botero, Darío Indalecio. Descentralización y Neoliberalismo, balance de un proceso. Bogotá D.C.: Fondo Editorial, CEIR- Realidad Municipal, 1992.

Sánchez, Ángel Ricardo. El poder y las constituyentes en la historia colombiana 1886-1992. Constitución, gobernabilidad y poder. Universidad Nacional de Colombia/ Universidad Industrial de Santander, 1996.

Uprimny Yepes, Rodrigo; Rodríguez Villabona, Andrés Abel. Libro interpretación judicial, módulo de autoformación. Bogotá D.C.: Consejo Superior de la Judicatura, Escuela Judicial “Rodrigo Lara Bonilla”, 2003.

Uprimny Yepes Rodrigo; Uprimny, Yepes Inés Margarita; Parra Vera, Oscar. Derechos humanos y derecho internacional humanitario módulo de autoformación, Bogotá D.C.: Fundación Social, Consejo Superior de la Judicatura, Escuela Judicial “Rodrigo Lara Bonilla”, 2006.

Vargas Velásquez, Alejo. La democracia en Colombia: al final del túnel o en la mitad del laberinto. Bogotá D.C.: Universidad de los Andes, 1993.

Younes Moreno, Diego. Derecho constitucional colombiano, Décimo Segunda Edición. Bogotá D.C.: Editorial Legis, 2012.

\section{JURISPRUDENCIA CONSULTADA}

Colombia, Corte Constitucional, Sentencia C- 157 de 1998, Magistrados Ponentes: Dr. Antonio Barrera Carbonell y Dr. Hernando Herrera Vergara.

Colombia, Corte Constitucional, Sentencia C- 1435 de 2000, con ponencia de la magistrada Cristina Pardo Schlesinger. Objeciones presidenciales al Proyecto de ley No. 118 de 1999 Cámara - 236 de 2000 Senado "Por la cual se modifica el inciso tercero del artículo 57 de la ley 30 de 1992".

Colombia, Corte Constitucional, Sentencia C-926 de 2005, Demanda de inconstitucionalidad contra el artículo 84 (parcial) de la Ley 812 de 2003, Magistrado Ponente: Dr. Jaime Córdoba Triviño.

Colombia, Corte Constitucional, Sentencia C-507 de 2008, Demanda de inconstitucionalidad contra los artículos 10, 27, 38 y 129 de la Ley 1151 de 2007 "por la cual se expide el Plan Nacional de Desarrollo 2006-2010", Magistrado Ponente: Dr. Jaime Córdoba Triviño.

Colombia, Corte Constitucional, Sentencia T-050 de 2013, Acción de tutela incoada mediante apoderado por Juan Manuel Latorre Carvajal, contra la Universidad Industrial de Santander, lección de Rector de la UIS por ser de carácter definitivo y tener otro mecanismo de defensa judicial.

Colombia, Corte Constitucional, Sentencia T-037 de 2012, acción de tutela instaurada por Leidy Daniela Vásquez Lentino contra la Universidad Manuela Beltrán y el Instituto Colombiano de Crédito y Estudios Técnicos en el Exterior - ICETEX, Magistrado Ponente: Luis Ernesto Vargas Silva. 
Colombia, Corte Constitucional, Sentencia T-544 de 2006, Acción de tutela instaurada por Hernando José Fernández Castilla contra IAFIC-Universidad Simón Bolívar y Katty Tinoco de Char. Magistrado Ponente: Dr. Alvaro Tafur Galvis.

Colombia, Corte Constitucional, Sentencia C-008 de 2001, Demanda de inconstitucionalidad contra el Artículo 103 de la Ley 30 de 1992, Actores: James Fernández Cardozo y Carlos Olmedo Arias Rey, Magistrado Ponente: Dr. Alvaro Tafur Galvis

Colombia, Corte Constitucional, Sentencia C-337 de 1996, Acción pública de inconstitucionalidad contra los artículos 28 y 29 (parciales) de la Ley No. 30 de 1992 "por la cual se organiza el servicio público de la Educación Superior". Materia: De la autonomía universitaria para admitir y seleccionar a sus alumnos. Actor: Alvaro Sanjuan Sanclemente Magistrado Ponente: Dr. Hernando Herrera Vergara.

Colombia, Corte Constitucional, Sentencia T-933 de 2005, Demandado: Universidad de Manizales, Magistrado Ponente: Dr. Rodrigo Escobar Gil

Colombia, Corte Constitucional, Sentencia T-691 de 2012, Acción de tutela presentada por Heiler Yesid Ledezma Leudo contra la Universidad Distrital Francisco José de Caldas - Facultad Tecnológica. Magistrada Ponente María Victoria Calle Correa. 